\title{
VACCINE DEVELOPMENT- A COMPLEX SCIENCE
}

\author{
Afifa Ansari ${ }^{1}$, Ayush Madan ${ }^{2 *}$ Divya Prakash ${ }^{3}$ \\ ${ }^{1,2,3}$ School of Biological Engineering \& Sciences, Shobhit University Gangoh, Saharanpur, \\ Uttar Pradesh, India-247341
}

*Corresponding Author : Ayush Madan

Article DOI: https://doi.org/10.36713/epra8007 DOI No: 10.36713/epra8007

\begin{abstract}
Several human vaccinations were created at the end of the nineteenth century, Smallpox, rabies, plague, cholera, and typhoid vaccinations are among them. Major things to acknowledge before vaccine preparation is that we have to isolate the antibody firstly which our body has produced against the pathogen and what is the composition of that antibody and also the structure of an antibody. $A$ vaccine is administered to induce immunity in an individual's body. Typically, the manufacture of vaccine uses viral or bacterial antigen in it. It may be killed or attenuated vaccine (live but less virulent). The foremost step to produce a vaccine is to select the strains for the vaccine and to culture the strain or microorganism. These two steps are collectively known as the upstream process. This is further followed by isolation and purification of the microorganism and then after the inactivation of the organism which is used for vaccine preparation formulation of vaccine begins. The last step is to check quality control and then further lot release; all of these steps are included in downstream processing. Mostly used vaccines lack efficiency, potency and safety. To take the vaccine preparation to next level, the introduction of monoclonal antibodies, recombinant DNA, and protein engineering have been accelerated knowledge of pathogenic mechanisms. This review provides a new approach to the development of the succeeding generation of vaccines.
\end{abstract}

\section{INTRODUCTION}

A vaccine is a medicine that is prepared to improve immunity against a specific pathogenic disease. To begin with we need to know about how our immunity works with the vaccine ${ }^{[1]}$. When pathogens or foreign particles such as germs, viruses, bacteria and fungi etc. enter our body, they multiply and attack our body and cause illness. To prevent the illness, our immune system uses its specialized cells say Leukocytes. Innate immunity and acquire immunity collectively work as the immune system. Vaccine boosts up immunity and helps in fighting the deadly pathogens. When inactivate or weaken disease-causing microorganisms enter the body, they initiate an immune response ${ }^{[2]}$. This response mimics the body's natural response to infection. Some vaccines are capable of providing a long-term response against the infection (Active immunization) and then some vaccines begin their work rapidly but are short-lasting (Passive immunization).

\section{IMMUNOLOGY BEHIND VACCINE DEVELOPMENT}

Even though this article is about vaccine development, however, we need to know about immunology to know about vaccines. Immunology is the study of the immune system of one individual's body and functions of the immune system and also its disorder [3]. The immune system provides immunity to particular diseases caused by pathogens through antibodies ${ }^{[4]}$. Antibodies are proteins that are released by our body's immune system to break down or neutralize the toxins released by the diseasecausing microorganism or pathogen Antigens are foreign substances which cause the production of antibody. So, antibodies are disease-specific, which implies that only a type of antibody works against a 
specific type of antigen ${ }^{[5]}$. For example, Measles antibody only protects from measles disease and will have no other effects on mumps or rabies disease ${ }^{[6]}$. Immunity is classified into two- ACTIVE and PASSIVE.

If we talk about active immunity, it protects our body when it comes to exposure to a diseasecausing microorganism say pathogen. Active immunity starts producing antibodies against the pathogen. Active immunity provides immunological memory, which means it will protect the body if it comes in contact with the disease in future, the immune system will recognize the pathogen and produce the antibody which is needed. On top of that, it is long-lasting ${ }^{[7]}$.

Passive immunity can be natural or artificial depending on the circumstances. In passive immunity, one person is administered antibodies against the infection rather than producing them through their immune system. For instance, a mother's placenta helps in providing immunity to a newborn baby (Passive immunity). Passive immunity begins to work very rapidly. So, if one needs immediate protection from a specific disease, passive immunization can be helpful. Tetanus or other antitoxins are examples of passive immunization. A disadvantage of passive immunity is that it only lasts for a few weeks or months ${ }^{[8]}$.

\section{STAGES OF VACCINE DEVELOPMENT}

A vaccine is a preparation that protects by forming antibodies and by boosting the immunity against the infection or disease. It is prepared from the weakened or killed version of the causative agent of disease it is administered to induce immunity. Vaccination is a process by which immunity to a pathogen is elicited (meaning that one who has taken the vaccine have been developed antibodies against the pathogen and now is immune). Nowadays vaccines are being developed with genetic engineering, it lets not cause any infection while it enters the body. So, the stages involved in vaccine production are-

To identify the strains for vaccine production:
a. Proliferation of microorganisms.
b. Isolation and purification of microorganism
c. Inactivation of microorganism
d. Formulation of vaccine
e. Quality control and release

a. To identify the strains for vaccine productionThe process begins with small amounts of a specific virus (seed). Viruses or bacteria used in the manufacturing process must be derived from a Seed Lot System. Each Seed Lot should have a record of its origin, including purification history and storage conditions ${ }^{[9]}$. Impurities, such as other similar viruses and even mutations of the same type of virus, must be removed from the virus ${ }^{[10]}$. The virus must be kept under "perfect" conditions, which usually means frozen, to prevent it from becoming stronger or weaker than desired ${ }^{[11]}$. The seed is chosen based on several parameters, including the efficiency of the eventual vaccine as well as its side effects ${ }^{[12]}$.

b. Proliferation of microorganisms- For culturing the bacteria there are a couple of methods, say batch culture or continuous culture. In batch culture, microbes grow in a close container and have very less or no contamination. If we talk about continuous culture, the microorganism is cultured in a tank that receives a new medium regularly and removes used media regularly ${ }^{[13]}$. Alternate methods uses in the growth of microorganisms are cell culture, bird embryos, live animal inoculation and transgenic animals ${ }^{[14]}$.

c. Isolation and purification of microorganismsIn the manufacture of vaccines, components whose qualities differ significantly from those of the target result are removed. Isolation and purification can be accomplished using a variety of techniques like chromatography, centrifugation, and filtration ${ }^{[15]}$. Chromatography is a class of physical separation techniques defined by the separation of mixtures due to changes in sample component distribution coefficients between two phases, one stationary and the other movement. Example: Modified Vaccinia Ankara virus (Smallpox vaccine) ${ }^{[16]}$. Centrifugation is a method of sedimentation and separation of solid particles from liquids that uses centrifugal force as a driving factor. It is used to separate and purify pathogenic virus antigens as well as other agents utilized in vaccine manufacturing. Example: Influenza vaccine, rabies vaccine, Hepatitis $B$ vaccine, and Japanese encephalitis vaccine production ${ }^{[17]}$. Filtration is the separation of particles from a liquid by forcing the solution through a filter by applying pressure to the solution ${ }^{[18]}$.

d. Inactivation of microorganisms- To make an inactivated or killed vaccine there's the necessity to inactivate the microorganism. This sort of vaccine is made by inactivating a pathogen with heat or chemicals like formaldehyde or formalin. The pathogen's ability to multiply is destroyed, but it remains "intact" so that the immune system can 
recognize it (Virus vaccines of this type are usually referred to as "inactivated," rather than "killed," because viruses aren't considered alive) [19]

e. Formulation of vaccine- A vaccination contains additional substances in addition to microorganisms or parts of microorganisms. Suspending fluids, a liquid that contains the chemicals used in the manufacturing of vaccinations to kill or weaken the organism. Sterile water, saline, or protein-containing fluids ${ }^{[20]}$. Influenza and yellow fever vaccines, which are made from chicken eggs, contain egg proteins. Hepatitis B vaccines are manufactured by transfecting cells of Saccharomyces cerevisiae (baker's yeast) with the gene encoding the hepatitis B surface antigen, and residual amounts of yeast proteins are found in the final product [21]. Preservative and stabilisers like Albumin, Phenols, Glycine, Monosodium glutamate (MSG), and 2phenoxy-ethanol, which are used as stabilisers in a few vaccines to help the vaccine remain unchanged when exposed to heat, light, acidity, or humidity ${ }^{[22]}$.

f. Quality control and release- Quality control for new vaccinations are critical since we must confirm that the product is safe, effective, and pure as well as pass the stability test. Once immunizations are initiated, national authorities and WHO carefully analyse and determine the severity of any potentially harmful side effects and responses from vaccine recipients ${ }^{[23]}$.

\section{TYPES OF VACCINES}

Vaccines are made up of either the full diseasecausing organism or a portion of it, a microbe or some of its constituents. Vaccines could be created in a variety of ways ${ }^{[24]}$.
a. Live-attenuated
b. Inactivated
c. Recombinant sub-unit
d. Toxoid

a. Live-attenuated vaccine- Live vaccinations employ a weakened (or attenuated) version of the disease-causing bacterium. These vaccines elicit a powerful and long-lasting immune response because they are so identical to the natural infection they help prevent. Most live vaccinations can provide lifetime protection against a germ and the disease it causes with just one or two doses ${ }^{[25]}$. Currently, a variety of live attenuated vaccines are in use. For instance, measles, polio (Sabin vaccine), rotavirus, smallpox, tuberculosis, varicellazoster (chickenpox), yellow fever ${ }^{[26]}$.

b. Inactivated- An inactivated vaccination employs a dead virus or bacterium to boost the immune system and protect the body from illness. Because the bacteria or virus is no longer alive, it can no longer replicate or cause disease [27]. The US Food and Drug Administration has authorised 85 single and combination vaccinations for use (FDA) ${ }^{[28]}$. Some examples include hepatitis A, influenza, Japanese encephalitis, rabies, typhoid etc. ${ }^{[27]}$

c. Recombinant sub-unit- Recombinant subunit vaccines are also known as recombinant protein vaccination are manufactured utilizing protein antigens. The method employed in the formulation of this sort of vaccination is recombinant DNA technology. Recombinant DNA methods have a lot of promise for developing low-cost, safe, and effective vaccinations. Two categories of recombinant vaccines are established. One is vectored and the other is a Recombinant subunit, produced by a heterogeneous protein expression system [29-30].

d. Toxoid- A toxin-based vaccination that has been kept neutral while still eliciting an immunological response to the toxin ${ }^{[31]}$. The poison enters the circulation and causes the disease's symptoms. To induce immunity, the protein-based toxin is made harmless and utilized as an antigen in a vaccine. The toxoid is adsorbed to aluminium or calcium salts, which act as adjuvants, to enhance the immunological response ${ }^{[31]}$. ExamplesTetanus toxoid, diphtheria toxoid ${ }^{[31]}$.

\section{NOVEL APPROACHES TO THE VACCINE}

Some several techniques and hypotheses outline the new vaccines approach. According to immunokinetics, the strength of the immune response increases as the speed of the above contact or release increases, and decreases as the speed of the above contact or release decreases with a reduction in it ${ }^{[32]}$. Experimental data is used to support clonal selection and idiotypic immune network theories ${ }^{[32]}$. The immunokinetics theory is mostly confirmed by natural events that everyone can observe and verify 
[32). It accounts for the fact that diseases that evolve quickly, such as smallpox, generate a greater immune response ${ }^{[32]}$. The research found that sublingual delivery resulted in increased bioavailability of almost all vaccine compositions ${ }^{[32]}$. As a result, it seems to be a viable method for testing the immunokinetics working hypothesis in action ${ }^{[32]}$. A novel vaccination that is administered sublingually might increase the efficacy, practicality, and safety of existing vaccines ${ }^{[32]}$. Vaccines may also trigger an immune response to cancer, therefore weakening autoimmune diseases ${ }^{[32]}$. Recombinant DNA technology, monoclonal antibodies, and protein engineering are the prime illustrations of novel approaches to the next generation of vaccines ${ }^{[2]}$. Recombinant DNA and protein engineering technologies are referred to as biotechnology ${ }^{[2]}$.

\section{CONCLUSION}

People are afraid to take vaccinations during the COVID-19 pandemic because of misconceptions and rumours that immunisation would cause disease and harm to everybody. So, one must discover what necessity vaccination is, what it does to our body, and most importantly, that it does not affect the body. There are a few cases in a billion when vaccines produce adverse side effects, but they usually fade away with time. The stages of vaccine development are a quick topic to learn about, and the most intriguing aspect is the different types of vaccines that exist presently. The stages of vaccine development are a comprehensive topic to study, and the most intriguing aspect is the variety of vaccines that exist now. Vaccine preparation is complicated, yet it couldn't be more fascinating to study. With the advancement of technology, new and improved vaccination methods are being developed to make them more effective.

\section{REFERENCES}

1. Offit, P. (2018). Vaccine Development, Testing, and Regulation | History of Vaccines. [online] Historyofvaccines.org. Available at: https://www.historyofvaccines.org/content/article s/vaccine-development-testing- and-regulation.

2. (PDF) Current approaches to vaccine preparation. (2016, February 5). Retrieved from ResearchGate.

3-8. Immunology and Serology. (2019). Retrieved from www.hopkinsmeicine.org website: https://www.hopkinsmedicine.org/health/ treatmenttests-and-therapies/immunolo gy-and-serology

9-22. Vaidhyanathan, B., \& Biotechnology, $Y$. (n.d.). Vaccine Production technique, growing the microorganisms in maximum titre. Retrieved from website: https://labmonk.com/notes/wpcontent/u ploads/2019/10/vaccineproductiontechni ques.pdf

23. Manufacturing, safety and quality control of vaccines. (2020, December 8). Retrieved from www.who.int website: https://www.who.int/newsroom/feature- stories/detail/manufacturingsafety-and-quality-control

24. Megan, W. (2012). Pharma vaccine fact book ( $p$. 107). Retrieved from https://issuu.com/meganwaters/docs/phr ma_final_eng_rgb (Original work published 2014)

25. Policy (OIDP), O. of I. D. and H. (2021, April 26). Vaccine Types. Retrieved from HHS.govwebsite: https://www.hhs.gov/immunization/basic s/types/index.html

26. Live Attenuated Vaccines. (n.d.). Retrieved July 10, 2021, from globalhealthprimer.emory.edu website: http://globalhealthprimer.emory.edu/targ etstechnologies/live-attenuated-vaccine s.html

27. pat, B. (2021, May 21). How inactivated vaccines help your body fight a virus. Retrieved June 27, 2020, from Very well Health website:

https://www.verywellhealth.com/what-is- aninactivated-vaccine-201081

28. Center for Biologics Evaluation and Research. (2019). Vaccines Licensed for Use in the US. Retrieved from U.S. Food and Drug Administration website: https://www.fda.gov/vaccines-blood-biol ogics/vaccines/vaccines-licensed-use-u nitedstates

29. Clark, T. G., \& Cassidy-Hanley, D. (2005). Recombinant subunit vaccines: potentialsandconstraints. Developments in Biologicals, 121(2005;121:153-63.), 153-163. Retrieved from https://pubmed.ncbi.nlm.nih.gov/159624 78/

30. Recombinant Subunit

Sartorius Vaccines.(n.d.).Retrieved from https://www.sartorius.com/en/application s/biopharmaceutical-manufacturing/vacc ines/vaccine-development/recombinant- subunitvaccines

31. MODULE 2 - Toxoid vaccines - WHO Vaccine Safety Basics. (2019). Retrieved from Vaccine-safety-training.org website: https://vaccine-safety-training.org/toxoidvaccines.html

32. Silvestrini, B. (2013). Immunokinetics: a new approach to vaccines. A working hypothesis. Ann Ist Super Sanità, 49(3), 306-308. https://doi.org/10.4415/ANN_13_03_12 